\title{
Parton distributions from lattice QCD: an update
}

\author{
W. Detmold ${ }^{a}$, W. Melnitchouk ${ }^{b}$ and A. W. Thomas ${ }^{c}$ \\ a Department of Physics, University of Washington, Box 351560, Seattle, WA 98195, U.S.A. \\ ${ }^{b}$ Jefferson Lab, 12000 Jefferson Avenue, Newport News, VA 23606, U.S.A. \\ 'Special Research Centre for the Subatomic Structure of Matter, and Department of Physics and \\ Mathematical Physics, University of Adelaide, 5005, Australia.
}

We review the extraction of parton distributions from their moments calculated in lattice QCD, focusing in particular on their extrapolation to the physical region. As examples, we consider both the unpolarized and polarized isovector parton distributions of the nucleon.

\section{HADRON STRUCTURE FROM LAT- TICE SIMULATIONS}

One of the defining features of any hadron are its quark and gluon (parton) momentum distributions. Considerable information has been accumulated on parton distribution functions (PDFs) of the nucleon and nuclei from deep inelastic scattering and other high energy experiments over the past 3 decades.

Theoretically, the operator product expansion (OPE) in QCD allows one to isolate the soft, nonperturbative PDFs from the hard, perturbatively calculable scattering processes. The only rigorous theoretical approach currently capable of calculating observables in the non-perturbative regime from first principles is lattice QCD. Since the numerical simulations are performed in Euclidean space-time, however, it is not possible to compute the PDFs directly as a function of the light-cone momentum fraction, $x$. Instead, the OPE formally expresses the moments of PDFs in terms of matrix elements of local twist-2 operators, $O^{(n)}$, between hadron states which can be calculated on the lattice [1]. For a generic parton distribution $q(x)$ in a hadron $H$, one has

$$
\left\langle H\left|O^{(n)}\right| H\right\rangle \stackrel{\text { OPE }}{\sim}\left\langle x^{n}\right\rangle_{q} \equiv \int_{-1}^{1} d x x^{n} q(x) .
$$

Over the last two decades, significant effort has been invested in lattice calculation of the mo- ments of various PDFs [2-6]. Despite the impressive progress, several approximations are still necessary in order to relate the lattice calculations to experiment. Firstly, the results at some finite lattice spacing, $a$, must be extrapolated to the continuum limit, $a \rightarrow 0$. The finite number of lattice sites also means that an extrapolation to the infinite volume limit is necessary to include important physics arising from the longrange part of the nucleon wave function. In addition, since the computational cost of simulations scales with the quark mass roughly as $m_{q}^{-4}$, current lattice simulations are performed with unphysically large masses for $u$ and $d$ quarks, typically $m_{q}^{\text {latt }}>30 \mathrm{MeV}$, so that an extrapolation to physical masses, $m_{q}^{\text {phys }} \approx 5 \mathrm{MeV}$, is essential [7].

In this paper we focus on the chiral $\left(m_{q}\right)$ extrapolation, which is found to play a particularly vital role in understanding the connection between the lattice results and phenomenology. Whereas the lattice simulations yield results $[3,4]$ for the moments of unpolarized quark distributions in the nucleon which are typically $50 \%$ larger than experiment when extrapolated linearly to $m_{q}^{\text {phys }}$, inclusion of the non-linear dependence on $m_{q}$ arising from the long-range structure of the nucleon removes most of the discrepancy [8]. A corresponding analysis of moments of the PDFs in the pion was performed in Ref. [9]. 


\section{CHIRAL SYMMETRY AND LAT- TICE QCD}

The importance of chiral symmetry and the role of the pion cloud in hadronic physics is well known $[10,11]$. At small quark masses, hadronic observables can be systematically expanded in a series in $m_{q}$ within the framework of chiral perturbation theory ( $\chi \mathrm{PT})$. While the expansion coefficients are generally free parameters, one of the unique consequences of spontaneous chiral symmetry breaking in QCD is the appearance of terms involving odd powers or logarithms of $m_{\pi}$. From the Gell-Mann-Oakes-Renner relation, which relates the quark and pion masses at small $m_{\pi}, m_{\pi}^{2} \sim m_{q}$, one finds that such terms are non-analytic in the quark mass. Furthermore, their coefficients, which are determined from the infrared behavior of the pion loops, are generally model independent.

The lattice moments in general receive contributions from diagrams in which the local operator insertions are on quark lines which are connected to the nucleon source, as well as those where the operator acts on a quark loop in the vacuum, which is connected only by gluon lines to the quark lines originating in the nucleon source. Obtaining a signal from the latter, "disconnected graphs" is extremely difficult on the lattice, and to date only the connected contributions to the lattice PDF moments have been computed. Fortunately, the near degeneracy of the $u$ and $d$ quark masses means that the disconnected diagrams cancel in the isovector difference $u-d$ of PDFs, allowing an unambiguous comparison of the connected isovector PDF lattice moments with experiment.

The mass dependence of the moments of the unpolarized isovector PDF of the nucleon can be parameterized as $[8,12-14]$

$$
\begin{aligned}
\left\langle x^{n}\right\rangle_{u-d} & =a_{n}\left(1+c_{\mathrm{LNA}} m_{\pi}^{2} \log \frac{m_{\pi}^{2}}{m_{\pi}^{2}+\mu^{2}}\right) \\
& +b_{n} \frac{m_{\pi}^{2}}{m_{\pi}^{2}+m_{b, n}^{2}},
\end{aligned}
$$

where (for $n>0$ ) the chiral coefficient is given by [15] $c_{\mathrm{LNA}}=-\left(1+3 g_{A}^{2}\right) /\left(4 \pi f_{\pi}\right)^{2}$. The $n=0 \mathrm{mo}$ ment, which corresponds to isospin charge, is not renormalized by pion loops. The argument of the logarithm corresponds to the case where the pion loops are regulated with a simple, sharp threemomentum cut-off. The parameter $\mu$ has the effect of suppressing the rapid variation of the chiral logarithm away from the chiral limit. Physically it is related to the size of the nucleon core, which acts as the source of the pion field [11]. Since the isovector distribution $u(x)-d(x) \rightarrow \delta(x-1 / 3)$ in the heavy quark limit (i.e., as $m_{q} \rightarrow \infty$ ), one may choose to constrain the constant $b_{n}$ in Eq. (2) by $b_{n}=1 / 3^{n}-a_{n}\left(1-\mu^{2} c_{\mathrm{LNA}}\right)$. Using Eq. (2), the results for the $n=1,2$ and 3 moments of the unpolarized $u-d$ distribution with $\mu=500 \mathrm{MeV}$ are found to be in excellent agreement with the phenomenological values within the present errors [8].

A similar analysis leads to analogous lowest order LNA parameterizations of the mass dependence of the spin-dependent helicity and transversity moments $[12,16]$. The $n=0$ moment of the spin-dependent isovector PDF is equivalent to the axial charge of the nucleon, $g_{A}$. It is well known from the Adler-Weisberger sum rule that the $\Delta$ resonance plays a key role in $g_{A}$. Although not leading non-analytic, the $\Delta$ contribution to spin-dependent matrix element renormalizations is large and extremely important in countering the effect of wave function renormalization [12]. The tendency for cancellation between wave function and polarized vertex corrections means that, once virtual $\pi \Delta$ loops are included, the chiral variation as $m_{\pi} \rightarrow 0$ is approximately linear.

The curvature in the extrapolated lowest moments of the helicity distribution $\left(g_{A}\right)$ increases somewhat the discrepancy between the lattice value and experiment, although the contributions of the $\Delta$ largely reduce this effect $[12,16]$. Nevertheless, there does appear to be a residual $10-15 \%$ underestimation of $g_{A}$, while at the same time the $n=1$ and 2 moments of $\Delta u-\Delta d$ are somewhat overestimated (although the errors on the $n=2$ lattice data in particular are rather large).

The RBCK Collaboration has in fact observed an unusually strong dependence of $g_{A}$ on the lattice volume [6]. The effect of a finite lattice volume on the chiral loops can be estimated by replacing the continuum momentum integrals in the 
chiral expansion by finite sums over the allowed momenta on the lattice - as already done for baryon and vector meson masses [17]. Alternatively, one can estimate the effect very simply by replacing the factor $m_{\pi}^{2} /\left(m_{\pi}^{2}+\mu^{2}\right)$ in Eq. (2) by $\left(m_{\pi}^{2}+\Lambda^{2}\right) /\left(m_{\pi}^{2}+\mu^{2}\right)$, where $\Lambda=2 \pi / N a \equiv 2 \pi / L$ is the lowest non-zero momentum available to a pion on the lattice, with $N$ the number of lattice sites in each spatial dimension. The results of this simple calculation indicate that one may need a box $L \sim 4 \mathrm{fm}$ on a side to see substantial chiral curvature in the extrapolation.

\section{BJORKEN- $x$ DEPENDENCE OF QUARK DISTRIBUTIONS}

Although the Mellin transform (1) has a mathematically well-defined inverse, the inversion requires knowledge of the behavior of the moments along a contour in the complex- $n$ plane. Clearly, evaluation of nucleon matrix elements of twist-2 operators (on the lattice or elsewhere) cannot provide this - through the $\mathrm{OPE}$, appropriate matrix elements determine moments only for real, integer $n$. To proceed one must therefore assume a parametric structure of the underlying parton distribution. In parameterizations of PDFs from experimental data, quark distribution functions $f(x)$ (polarized and unpolarized) are often parameterized with the form

$x f(x)=A x^{b}(1-x)^{c}(1+\epsilon \sqrt{x}+\gamma x)$.

The parameters $b$ and $c$ determine the small- and large- $x$ behavior, respectively, and have physical interpretations in terms of Regge behavior and counting rules, whereas $\epsilon$ and $\gamma$ are introduced to provide additional freedom. Given this parametric form, one can use the various sets of moments extrapolated from the lattice data to fit the parameters in Eq. (3). Since there are at most three nontrivial moments available for each distribution, one must first reduce the number of free parameters. We choose to fit $\epsilon$ and $\gamma$ to an average of the available unpolarized and phenomenological parameterizations [18]. This leaves only the parameters $A, b$ and $c$ to be determined from the lattice data.

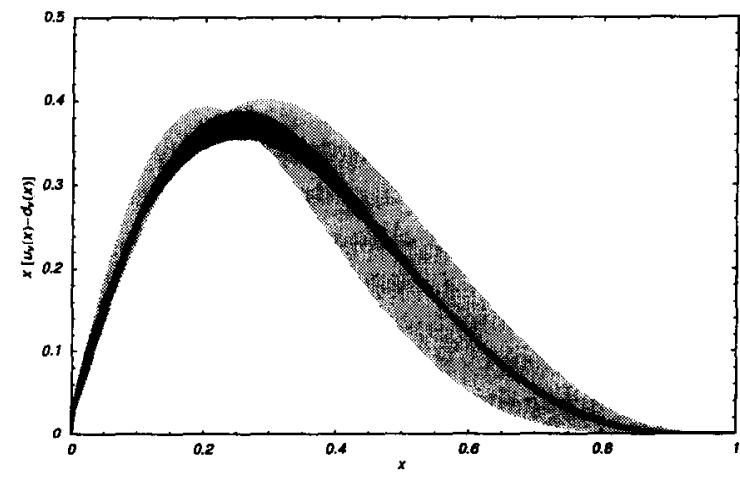

Figure 1. Reconstructed isovector valence quark distribution $x\left(u_{v}-d_{v}\right)$ in the proton at $Q^{2}=$ $4 \mathrm{GeV}^{2}$. The central fit curve (solid line) and error band (lightly shaded) are compared with the envelope of the phenomenological distributions (darkly shaded).

The unpolarized valence isovector distribution reconstructed [13] from the lattice moments is shown in Fig. 1 (solid line). To recover the valence moments, the relevant extrapolated lattice moments have been shifted by the corresponding moments of the sea quark distribution. In practice, since sea quarks are concentrated at small $x$, it is only the lowest moments ( $n=0$ in the polarized case, $n=1$ for unpolarized) that shift to any appreciable degree.

To present realistic error bands on the reconstructed distribution, a large ensemble $(\sim 200 \mathrm{el}-$ ements) of sets of moments is generated by randomly varying each moment within the extrapolated lattice error bars. The lightly shaded region then corresponds to the envelope of these distributions. The parameter values and errors are quoted in Table 1 and are given by the mean and standard deviation over the ensemble. The darkly shaded region corresponds to the range of (next-to-leading order) PDF parameterizations of $u_{v}(x)-d_{v}(x)$ at $Q^{2}=4 \mathrm{GeV}^{2}$. The agreement between the reconstructed distributions and the phenomenological parameterizations is clearly excellent. 
Table 1

Fit parameters for the reconstructed distributions. For each polarized data set, the upper (lower) rows show the parameters for fits with $b$ (c) fixed to its phenomenological value. In the unpolarized (polarized) fits, $\epsilon=1.96$ and $\gamma=29.1$ $(\epsilon=-0.63$ and $\gamma=10.1)$ were set to their phenomenological values.

\begin{tabular}{|c|ccc|}
\hline \hline Data set & $A$ & $b$ & $c$ \\
\hline \hline Unpolarized & $0.21(5)$ & $-0.63(3)$ & $3.8(6)$ \\
\hline Polarized, set I & $0.47(3)$ & -0.51 & $2.1(2)$ \\
& $1.9(2)$ & $-0.05(7)$ & 3.69 \\
\hline Polarized, set II & $0.28(5)$ & -0.51 & $1.4(2)$ \\
& $5(2)$ & $0.8(5)$ & 3.69 \\
\hline Polarized, set III & $0.61(4)$ & -0.51 & $2.5(1)$ \\
& $1.5(1)$ & $-0.24(6)$ & 3.69 \\
\hline Polarized, set IV & $0.70(5)$ & -0.51 & $3.0(2)$ \\
& $1.1(1)$ & $-0.39(5)$ & 3.69 \\
\hline \hline
\end{tabular}

In contrast to the unpolarized distribution, there are a number of issues that complicate the analysis of the polarized distributions. Firstly, from the crossing symmetry properties of spindependent structure functions, the $n=0$ moments extracted from the lattice correspond to moments of the total distribution, $\Delta q(x)+\Delta \bar{q}(x)$, and in order to construct the valence moment one must subtract (twice) the polarized sea moment. Unfortunately, little experimental information exists on $\left\langle x^{0}\right\rangle_{\Delta \bar{u}-\Delta \bar{d}}$. Consequently, we shall investigate two scenarios: $\left\langle x^{0}\right\rangle_{\Delta \bar{u}-\Delta \bar{d}}=0$ (as suggested in models in which the non-perturbative sea is generated through meson loops), and $\left\langle x^{0}\right\rangle_{\Delta \bar{u}-\Delta \bar{d}}=0.2(2)$ (as in quark models with $\mathrm{SU}(6)$ symmetry, when the Pauli exclusion principle is applied) - see Ref. [18] and references therein.

Secondly, as discussed above, finite volume effects have been found [6] to be particularly significant in lattice calculations of $g_{A}$, and to account for these we shall consider the effects of shifting the extrapolated moments towards their physical value. Finally, fitting the three parameters $A, b$ and $c$ with only three moments is unreliable (though possible), so one must presently set either $b$ or $c$ to its phenomenological value.
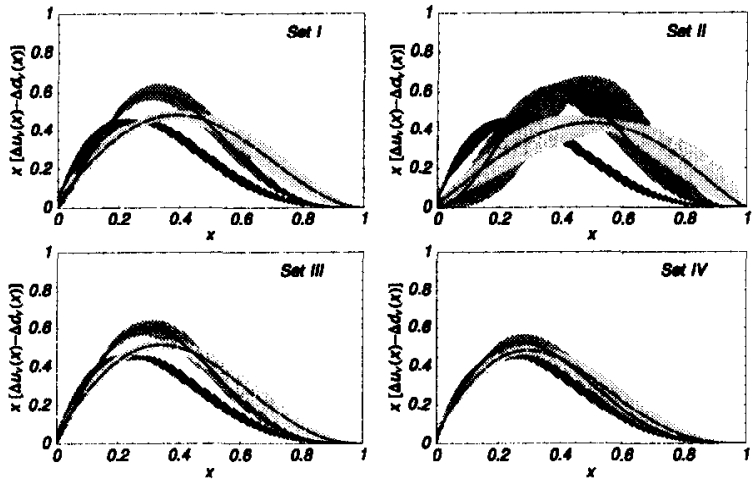

Figure 2. Reconstructed isovector valence helicity distribution, $x\left(\Delta u_{v}-\Delta d_{v}\right)$ at $Q^{2}=4 \mathrm{GeV}^{2}$. In each panel, the solid (dashed) curve and the corresponding error band is a best fit with $b(c)$ in Eq. (3) fixed, while the darkly shaded region corresponds to the envelope of the phenomenological distributions. The four sets of curves are described in the text.

In Fig. 2 we show the reconstructed PDFs for various shifting scenarios compared with phenomenological parameterizations. Each panel shows the fits and error bands with $b$ fixed (solid curve, medium shading), and with $c$ fixed (longdashed curve, light shading). The darkly shaded region corresponds to the envelope of the phenomenological distributions. In the upper left panel, the unmodified extrapolated lattice moments are used (data set I). Fits to these with either $b$ or $c$ fixed give a somewhat different reconstructed distribution, and the PDFs extracted from the lattice are not in agreement with the phenomenological fits [18].

In the upper right plot we show the resulting fits that arise from shifting the $n=0$ moment up by $0.2(2)$ to correct for the sea contribution which enters the lattice extrapolated moment (data set II). The agreement between the two fits (with $b$ and $c$ held fixed) is still poor and the errors are considerably increased. In the lower panels, we contemplate shifts of the moments to correct for possible finite volume (and other) effects in the lattice data and their extrapolations. On the left, 
we shift just the $n=0$ moment, $g_{A}$, so that it agrees with experiment (data set III), while on the right all three moments are shifted by the same relative percentage towards the experimental moments (data set IV). The latter is the best case scenario, with the agreement between the two reconstructed distributions significantly improved, and (not surprisingly) in excellent agreement with the phenomenological fits. The parameters for the various fits are given in Table 1.

\section{CONCLUSIONS}

The study of the PDFs of the nucleon using lattice QCD has already established several notable benchmarks. In the unpolarized case the level of agreement between the first three nontrivial moments of the isovector distribution and experiment is excellent, once the correct leading non-analytic behavior of chiral perturbation theory is incorporated into the extrapolation of the moments. For the polarized PDFs, the $\Delta$ isobar plays an important role, canceling much of the effect of the $\pi N$ loops, and giving rise to a significant reduction in the chiral curvature as $m_{\pi} \rightarrow 0$. For $g_{A}$ in particular, there is a rather unusual degree of sensitivity of the polarized moments to the volume of the lattice, and agreement with the experimental value improves as the volume increases [6].

Future lattice simulations need to focus both on accessing the small $m_{q}$ region, while simultaneously working on lattices of dimensions $L \sim 4 \mathrm{fm}$ (for pion masses $<300 \mathrm{MeV}$ ). The availability of higher moments would also be extremely valuable for determining the $x$ dependence of both the valence and sea quark distributions independently.

\section{REFERENCES}

1. M. Göckeler et al., Phys. Rev. D 54 (1996) 5705.

2. G. Martinelli and C. T. Sachrajda, Phys. Lett. B 196 (1987) 184; ibid B 217 (1989) 319.

3. D. Dolgov et al., Phys. Rev. D 66 (2002) 034506.

4. M. Göckeler et al., Phys. Rev. D 53 (1996) 2317; Nucl. Phys. Proc. Suppl. 53 (1997) 81;
Nucl. Phys. A711 (2002) 291; S. Capitani et al., Nucl. Phys. Proc. Suppl. 106 (2002) 299;

C. Best et al., hep-ph/9706502.

5. M. Fukugita et al., Phys. Rev. Lett. 75 (1995) 2092; S. Aoki et al., Phys. Rev. D 56 (1997) 433.

6. S. Sasaki, K. Orginos, S. Ohta and T. Blum, hep-lat/0306007; Nucl. Phys. Proc. Suppl. 106 (2002) 302.

7. A. W. Thomas, Nucl. Phys. Proc. Suppl. 119 (2003) 50.

8. W. Detmold et al., Phys. Rev. Lett. 87 (2001) 172001.

9. W. Detmold, W. Melnitchouk and A. W. Thomas, Phys. Rev. D 68 (2003) 034025.

10. A. W. Thomas, Adv. Nucl. Phys. 13 (1984) 1.

11. W. Detmold et al., Pramana 57 (2001) 251.

12. W. Detmold, W. Melnitchouk and A. W. Thomas, Phys. Rev. D 66 (2002) 054501 .

13. W. Detmold, W. Melnitchouk, and A. W. Thomas, Eur. Phys. J. direct C 3 (2001) 13.

14. A. W. Thomas, W. Melnitchouk and F. M. Steffens, Phys. Rev. Lett. 85 (2000) 2892.

15. D. Arndt and M. J. Savage, Nucl. Phys. A697 (2002) 429; J. W. Chen and X. Ji, Phys. Lett. B 523 (2001) 107.

16. W. Detmold, W. Melnitchouk and A. W. Thomas, Int. J. Mod. Phys. A 18 (2002) 1343; hep-lat/0210029.

17. R. D. Young et al., Phys. Rev. D 66 (2002) 094507; D. B. Leinweber et al., Phys. Rev. D 64 (2001) 094502.

18. W. Detmold, W. Melnitchouk and A. W. Thomas, Mod. Phys. Lett. A 18 (2003) 2681, hep-lat/0310003. 